\title{
Aerodynamic and Structural Evaluation of Horizontal Axis Wind Turbines with Rated Power over 1 MW
}

\author{
S. Laín ${ }^{1,2}$, B. Quintero ${ }^{1}$ and Y. López ${ }^{2}$ \\ ${ }^{1}$ Fluid Mechanics Research Group, phone: +57 2 3188000, e-mail: slain@uao.edu.co, bquintero@uao.edu.co \\ ${ }^{2}$ Energy Research Group (GIEN), phone: +57 2 3188000, e-mail: ylopez@uao.edu.co \\ Energetics and Mechanics Department, Universidad Autónoma de Occidente, \\ Calle 25 No 115-85, Km. 1 vía a Jamundí, Cali (Colombia)
}

\begin{abstract}
An aeromechanical evaluation of large (over 1 Mw of nominal power) Horizontal Axis Wind Turbines (HAWT's) is performed is this paper. The strategy is based on the combination of an aerodynamic module, which provides the three-dimensional pressure distribution on the HAWT's blades, an a structural module which takes such pressure forces as input data in order to compute both, blade deformation and strain and stress distributions over the blade.
\end{abstract}

The aerodynamic module combines the three-dimensional nonlinear lifting surface theory approach, which provides the effective incident velocity and angle of attack at each blade section, and a two-dimensional panel method for steady axisymmetric flow in order to obtain the 3D pressure distribution on the blade. Such pressure distribution constitutes the input data for the structural module, which is a finite element package whose output is the blade deformation and strain and stress distribution along the blade, as well as material induced fatigue.

This methodology is applied to study a $50 \mathrm{~m}$ long blade able to provide a nominal power of $3 \mathrm{Mw}$.

Key words: Wind turbine, aerodynamics, structural behaviour, numerical simulation, efficiency.

\section{Introduction}

Eventually, in normal running, the blades of a wind turbine must withstand the centrifugal forces, bending moments caused by the pressure aerodynamic forces and gyroscopic effects which appear during the orientation changes which can accompany gusts, for example. All of these phenomena imply the appearance of strain and stresses on the blades and also will induce material fatigue. Another potential risk is the appearance of aeroelastic instabilities which can even lead to catastrophic failure. In order to improve the design of HAWT's blades, all these effects have to be analysed. Very often simple explicit relations are used and, because the design must be at the safe side, all load calculations are set to their upper limits whereas high safety factors are added to guarantee the operation of the turbine over a long life time. Designs based on such simplified procedures, can become overdimensioned leading to high costs. Optimisation of the design requires more accurate estimations of the loads and therefore more elaborate models have to be used requiring computational tools that model the coupling of aerodynamics, structural dynamics and wind turbulence. Most of them were developed over the last few years at DEWI (Germany), ECN (Netherlands), FFA (Sweden), Garrad \& Hassan (United Kingdom) and RIS $\varnothing$ (Denmark). Their core is a conventional blade element aerodynamic model coupled with a beam structural model.

However, modern HAWT blades are composite thin structures with a rather sophisticated distribution of laminated resins spanwise and chordwise. Mechanical optimisation of such devices should be made by modelling the blade as a set of thin layer elements rather than using the beam elements approach. Accurate evaluation of interlaminar strengths require a detailed evaluation of the external force field, namely pressure field, which is supposed to supply a better estimate of rotor performance than simplified momentum theories, using global lift and drag forces at each section.

Following this line, a compromise between accuracy and computational cost has been found defining an enhanced non-linear Lifting Surface Method in order to compute pressure field on HAWT blades. The main features are: combined, 3D Interacting-twisted-Lifting Surface-/2D Vortex Panel Method; prescribed cylindrical wake with constant wake pitch relaxed iteratively; effective incident velocity and angle of attack at each section are found; pressure field is computed then over arbitrary profiles at non-stalled flow conditions as sequence of $2 \mathrm{D}$ pressure fields. In order to account for the fluid - structure interaction the commercial finite element package ANSYS has been used to evaluate the stress and strain distribution along the blade so the material induced fatigue or possible breakage can be estimated. In addition, the simulation allows to optimise the blade shape because the ratio weight - strength is a factor that defines the blade design. The validation of this strategy has been validated previously [1-2]. 
The interest of the present paper is focused on large wind turbines which are defined as those with nominal power output over $1 \mathrm{Mw}$. Figure 1 shows the growth of the rotor diameter with the generated power. Therefore, a key element in the blade construction is its weight, which is directly related with the fabrication costs and transport difficulties.

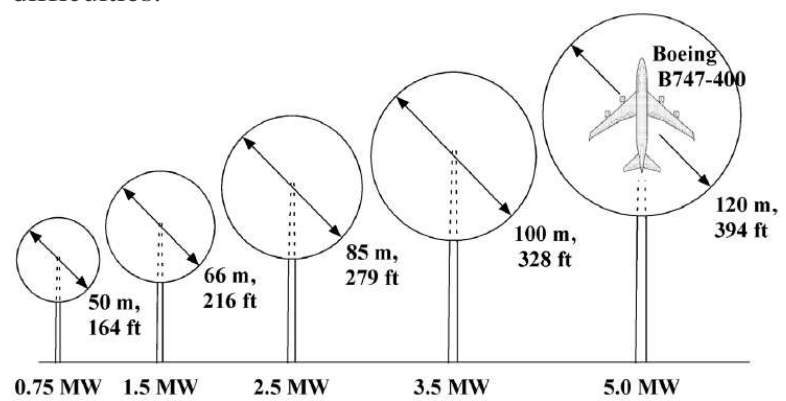

Fig. 1. Dependency of the rotor diameter with the generated power.

In the range of large wind turbines, from 30 to 70 meters, the blade weight grows as the cube of its length (Fig. 2) whilst the economic performance of the blades is inversely proportional to the specific weight, defined as the blade weight divided by the swept area $\left(\mathrm{kg} / \mathrm{m}^{2}\right)$. Following [3] increasing airfoil section thickness is a key tool in limiting blade weight and cost growth with scale. Thickened and truncated trailing edges in the inboard sections provide strong, positive effects on the blade structural performance. For a given rotor radius, in moving from the thin to thickest blade distribution the specific weight was reduced by $15 \%$, due to increased structural performance. The final conclusion, comparing the aerodynamic and structural studies, is that inboard sections should be weighted more strongly towards structural performance, while outboard sections are weighted most highly in aerodynamic characteristics.

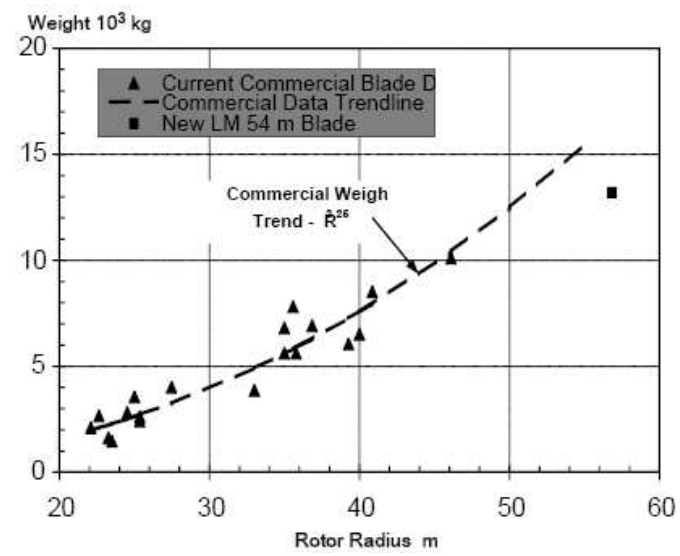

Fig. 2. Variation of the blade weight with rotor radius. Source: Composites Tech.

In this paper, the previously described strategy, namely the combination of the aerodynamic module, based on the Lifting Surface Method and the 2D panel method, and the structural module, based on the ANSYS finite element package, is applied to analyse the large HAWT considered in [3] from both, aerodynamic and structural points of view. This wind turbine has a blade of $50 \mathrm{~m}$ in length and a rated power of $3 \mathrm{Mw}$.

\section{Aerodynamic Analysis}

The considered configuration is the following: a steady, uniform wind flows over a HAWT with $\mathrm{K}$ blades of radius $\mathrm{R}$, rotating at constant angular velocity $\omega$ aligned with the incoming free stream velocity, $\mathrm{V}_{\infty}$. A cartesian system of coordinates $(\mathrm{X}, \mathrm{Y}, \mathrm{Z})$ is defined relative to the first blade (Fig. 3). Y direction coincides with the turbine rotation axis, oriented towards the incoming wind stream. $\mathrm{Z}$ axis goes along the blade span, oriented from root to tip and starting at hub pitching centre. $\mathrm{X}$ direction is the third orthogonal dextrogire axis lying on the rotation plane.

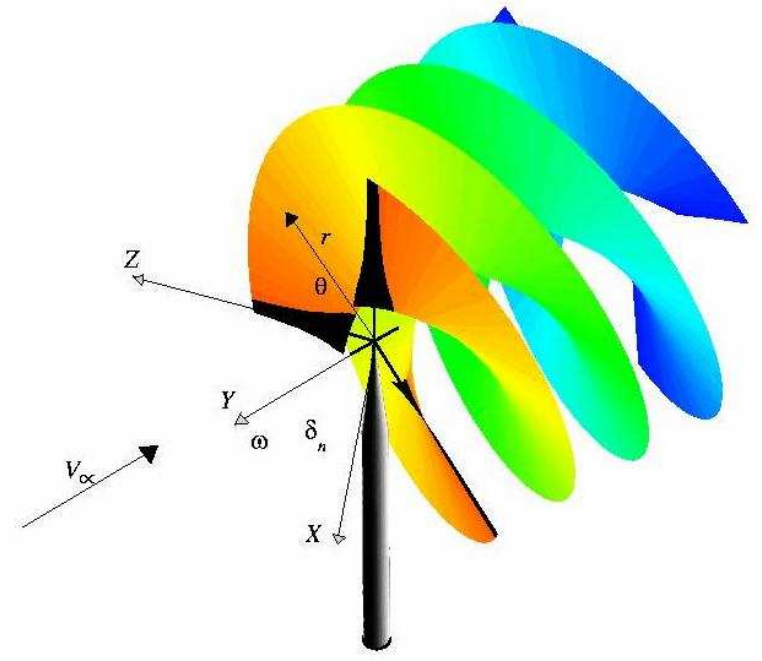

Fig. 3. Considered configuration of the wind turbine modelled by the Lifting Surface approach.

The absolute non-stationary irrotational velocity field is computed in terms of the upstream velocity, $\mathrm{V}_{\infty}$, plus a perturbation due to velocities induced by the vorticity field. It is assumed that vorticity is concentrated at thin blade boundary layers and wakes, modelled as vorticity sheets (Fig. 3). Vorticity associated to boundary layers can be merged into a single vortex surface, the Lifting Surface. Lifting Surface geometry is a cambered twisted continuous surface build up by the sequence of "profile cambered middle lines" going from the leading edge to the trailing edge [4]. This approach allows to obtain an effective velocity and angle of attack at each section of the blade [1].

The pressure distribution along the blade for non-stalled conditions is extracted from the effective relative velocity and angle of attack by means of a plane flow analysis for each section using a conventional $2 \mathrm{D}$ vortex panel method [5]. This pressure filed constitutes the input data for the structural module.

This methodology has been applied to the case presented in [3] based on a blade configuration of $50 \mathrm{~m}$ with a nominal power of $3.0 \mathrm{Mw}$. However, it is necessary to 
remark that, although [3] provides data about all the geometrical data (chord and pitch angle distribution along the span) the full details about the profile geometry and aerodynamic characteristics are not publicly available. Therefore, a detailed quantitative comparison with the data presented in [3] was not possible at this stage. The adopted approach has been to build the blade applying kinematic similarity with an old blade, ECOTECNIA 20/150, which is known to have a very good aerodynamic performance [1]. As that old blade is $10 \mathrm{~m}$ long, the first step was to multiply the geometrical dimension by 5 but maintaining the pitch angle and profile distributions. The kinematic similarity condition gives that the angular velocity should be $\omega=1.07 \mathrm{rad} / \mathrm{s}$. The basic design studied here, which is denoted as $\mathrm{W} 15 \mathrm{~S}$, is presented in Table 1.

TABLE 1.- Basic design of the W15S blade. $\omega=1.07$ $\mathrm{rad} / \mathrm{s}$

\begin{tabular}{rrrc}
\hline Radius [m] & Pitch [degrees] & Chord [m] & Profile \\
\hline 11.20 & 14.000 & 5.450 & Naca63221 \\
18.75 & 6.270 & 4.825 & Naca63221 \\
26.05 & 2.220 & 4.225 & Naca63221 \\
32.75 & 0.690 & 3.670 & Naca63218 \\
38.65 & 0.170 & 3.185 & Naca63218 \\
43.45 & 0.030 & 2.790 & Naca63215 \\
47.08 & 0.000 & 2.495 & Naca63215 \\
49.25 & 0.000 & 2.310 & Naca63215 \\
50.00 & 0.000 & 2.250 & Naca63215 \\
\hline
\end{tabular}

Several modifications were made to this basic configuration in order to evaluate its aerodynamic performance, which are listed in Table 2.

TABLE 2.- Study cases for the blade W15S

\begin{tabular}{cccc}
\hline Case & $\omega[\mathrm{rad} / \mathrm{s}]$ & $\begin{array}{c}\text { Chord \& pitch } \\
\text { distribution }[\mathrm{m}]\end{array}$ & Profiles \\
\hline Basic & 1.07 & Table 1 & Naca632XX \\
Basic & 1.07 & Table 1 & Naca632XX \\
Glauert & & Table 1 & Naca632XX \\
Case 1 & 1.45 & Table 3 & Naca632XX \\
Case 2 & 1.07 & & \\
\hline
\end{tabular}

TABLE 3.- Geometric parameters of the W15S blade considered in [3]

\begin{tabular}{rrr}
\hline Radius [m] & Pitch [degrees] & \multicolumn{1}{c}{ Chord [m] } \\
\hline 7.80 & 19.500 & 4.030 \\
13.00 & 13.000 & 4.472 \\
18.20 & 8.800 & 3.939 \\
23.40 & 6.200 & 3.452 \\
28.60 & 4.400 & 2.986 \\
33.80 & 3.100 & 2.534 \\
39.00 & 1.900 & 2.092 \\
44.20 & 0.800 & 1.659 \\
49.40 & 0.000 & 1.233 \\
\hline
\end{tabular}

Firstly, the performance of the Lifting Surface approach is compared versus the results of Glauert theory. Secondly, the angular speed is increased according to the data provided in [3]. Finally the chord and pitch angle distributions considered in [3] and references therein are considered. The power - incident velocity (top) and power coefficient - tip speed ratio (bottom) curves for those cases are shown in fig. 4 together with the computed data in [3].
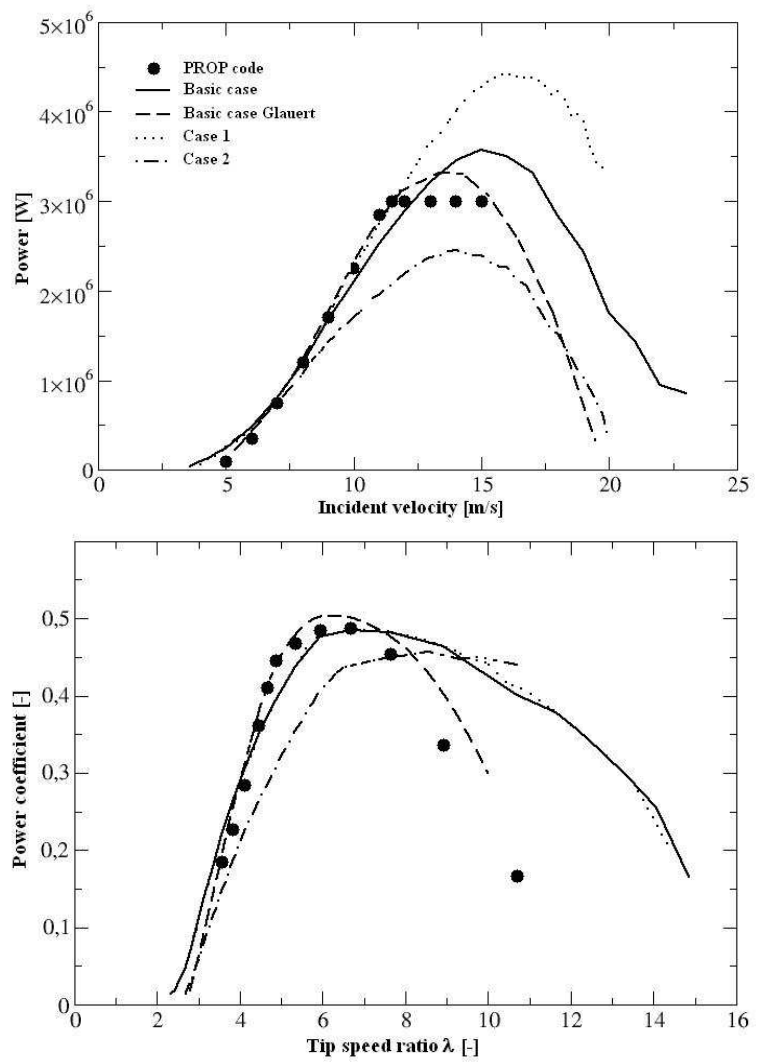

Fig. 4. Power - velocity (up) and power coefficient - tip speed ratio (down) curves for the W15S rotor.

In fig. 4 the points correspond to the computations presented in [3] with the so-called PROP code, which is based on the Glauert theory combined with the blade element theory. In such calculations it was assumed that the rotor diameter was $102 \mathrm{~m}$, a rated power of $3 \mathrm{Mw}$ and active power regulation. This is the reason why the power is kept constant after an incident velocity of $12 \mathrm{~m} / \mathrm{s}$. On the contrary, in the present computations the power regulation is due to aerodynamic stall leading to an absolute maximum in the power - velocity curve.

As it can be readily seen from fig. 4, the Basic Glauert case provides very similar results to those obtained with the PROP code in spite of the different geometric parameters. This is due to the fact that, in the Glauert theory, the influence of the profil geometry is very reduced. On the other hand, the Lifting Surface approach in the basic case provides the nominal power of $3 \mathrm{Mw}$ for an incident velocity slightly higher than $12 \mathrm{~m} / \mathrm{s}$ and also the maximum power coefficient is somewhat lower than 
that provided by the Glauert theory. This fact is consistent because the hypothesis involved in the Lifting Surface Method are less ideal that those in the Glauert theory. If the angular speed is increased, Case 1, the available power is increased but also the risk of turbine run away is substantially increased (dotted lines in fig. 4). Finally, Case 2 considers the reduction of the chord along the span which implies a reduction of the blade area and, therefore, also a reduction of the torque that the wind transfer to the blade. The final result is a significative decrement in both, the power and the power coefficient of the turbine.

\section{Structural Analysis}

As it has been previously mentioned, in normal running the blades of a wind turbine must withstand the centrifugal forces, bending moments caused by the pressure aerodynamic forces and gyroscopic effects which appear during the orientation changes which can accompany gusts, for example. All of these phenomena imply the appearance of strain and stresses on the blades and also will induce material fatigue leading, in some cases, to breakage. One phenomenon that cause it is aeroelastic instability which can be from several types, for instance, divergence o classical flutter. Divergence is a static phenomenon wherein the flow velocity (due to both, the ambient wind speed and the rotor rotation rate) becomes large enough so that the load produced for an incremental angle of attack change due to blade twisting is larger than the reaction load produced by the elastic restoring forces for the same amount of twist. The result is an effective loss of stiffness so the blade twists without bound, leading to catastrophic failure. Classical flutter is a dynamic phenomenon characterized by an interaction between blade bending and twisting oscillations. As the flow velocity increases, the aerodynamic loading causes the vibratory phase between these two motions to change, eventually leading to a negative damping situation and catastrophic failure. Both of these phenomena can occur at low angle of attack. A third type of aeroelastic instability is stall flutter. It is characterized by predominantly torsional blade oscillation and normally occurs at high angle of attack near stall. Therefore, in order to prevent these extreme cases and improve the design of HAWT's blades, at least some of these effects can be analysed with computational tools, which have the key advantage that they are cheap compared with the full scale measurements.

Following this line of reasoning, the aerodynamic module has been coupled with an structural module, where the pressure distribution along the blade and its geometry constitute the input data for the finite element code used to perform the structural calculation. The chosen finite element code has been the commercial package ANSYS due to its flexibility for handling information contained in files written in a more or less free format.

The geometry and the pressure data are contained in the aerodynamic output file, which are translated into the ANSYS data format by means of a simple FORTRAN routine. The geometric model of the W15S turbine is shown in fig. 5 .

According to the considerations presented in [3], the blade construction was assumed to be a stressed shell, which was composed of four primary components: a low pressure shell on the downwind side, a high-pressure shell on the upwind side and two shear webs bonded between the two shells (fig. 6). The considered composite material has been $65 \%$ polycarbonate and $35 \%$ fibreglass due to its high specific stiffness.

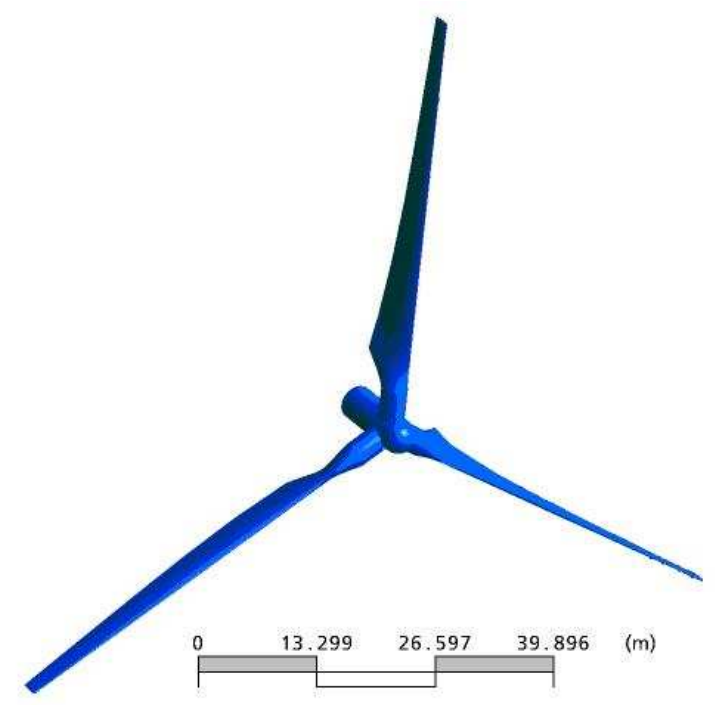

Fig. 5. Geometric model of the W15S blade.

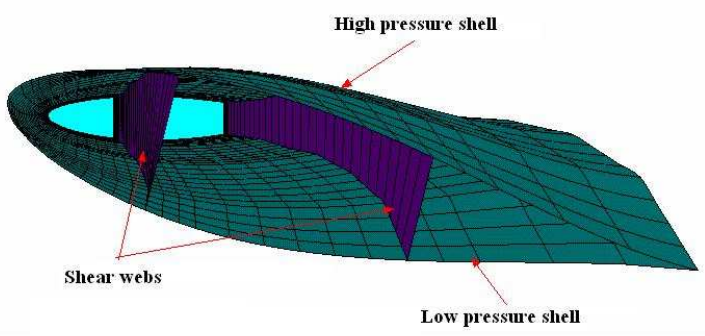

Fig. 6. Blade construction for the W15S blade.

The structural analysis for the W15S blade was performed for the basic case of Table 2 and has covered three aspects: static, dynamic and fatigue.

In the static analysis the blade deflection and the von Misses stresses were analysed. Nominal loads have been considered, i.e. the pressure loads acting on the blade at the point of maximum efficiency. In these conditions the maximum deflection obtained was $3.7 \mathrm{~m}$, an acceptable value because the tower clearance is around $10 \mathrm{~m}$. Fig. 7 shows the von Misses stresses for the high pressure shell (top) and shear webs (down). Consistently, the maximum stresses are located near the blade root and decrease towards the tip. Also, the Safety Factor was calculated in this case, providing values along the blade well above 1 which is the critical value. 

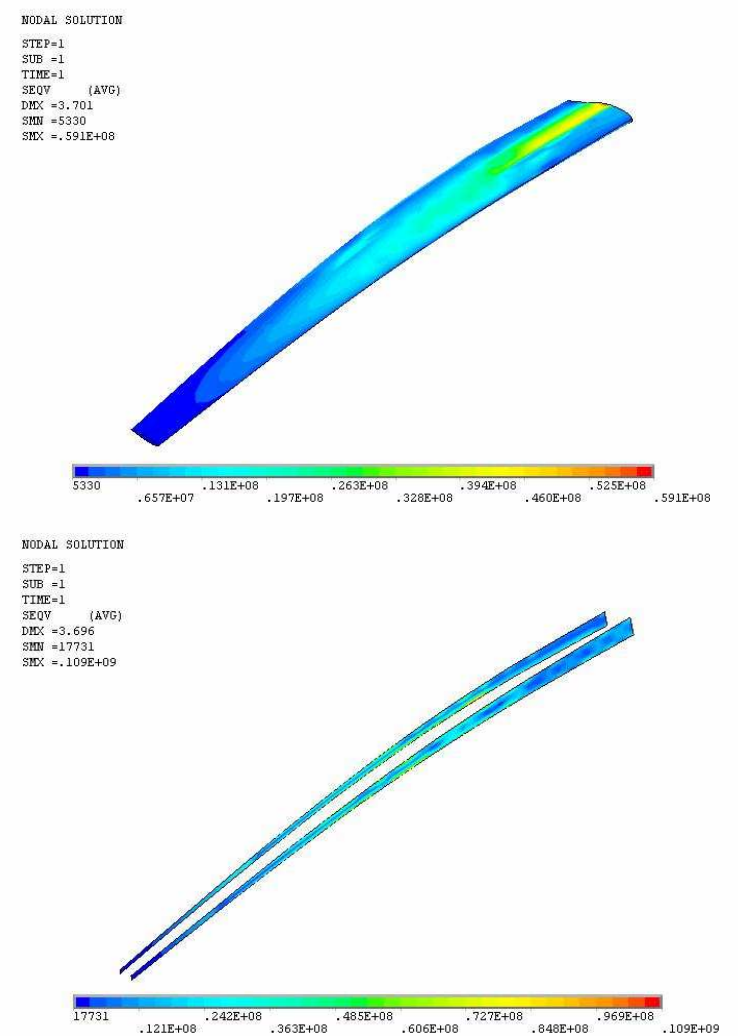

Fig. 7. Von Misses stresses in the high-pressure shell (up) and in the shear webs (down).

The dynamic analysis took into account the blade natural modes of vibration. Those must be uncoupled with the natural frequencies of the other components of the wind turbine in order to avoid mechanical resonance. The first four modes and the sixth were flection modes whilst the fifth was a torsion mode (fig. 8). The first natural frequency is $0.4 \mathrm{~Hz}$, a very similar value to those provided in [3].

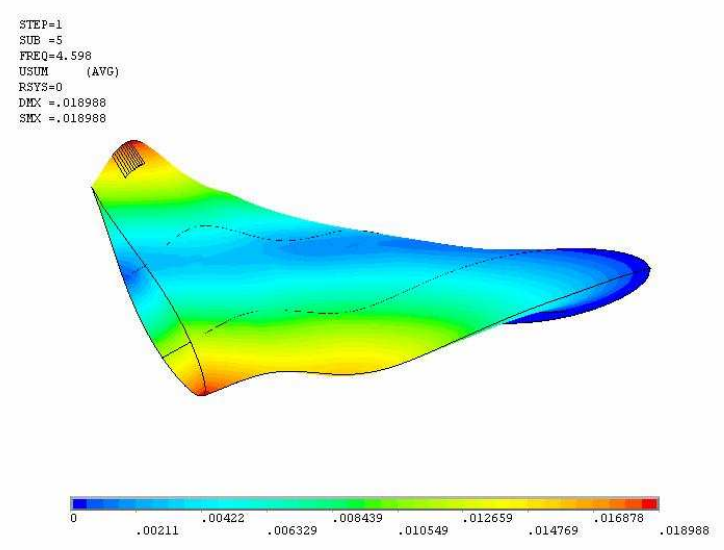

Fig. 8. Torsion mode of vibration of the W15S blade

In the fatigue analysis, the blade is put under the action of cyclic loads during a total time of 10 minutes in order to evaluate its accumulated damage. The loading is performed following the configuration shown in fig. 9. The model employed to evaluate the fatigue of a composite material has been the Degradation Lineal Accumulation model proposed by Palmgren [6] and Miner [7] which evaluate the accumulated damage at certain number of cycles. Fig. 10 shows the von Misses stresses along the blade after $330 \mathrm{~s}$ of cyclic loading.

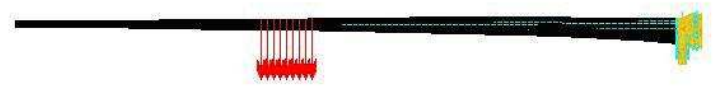

Fig. 9. Scheme of the cyclic loads applied in the fatigue analysis.

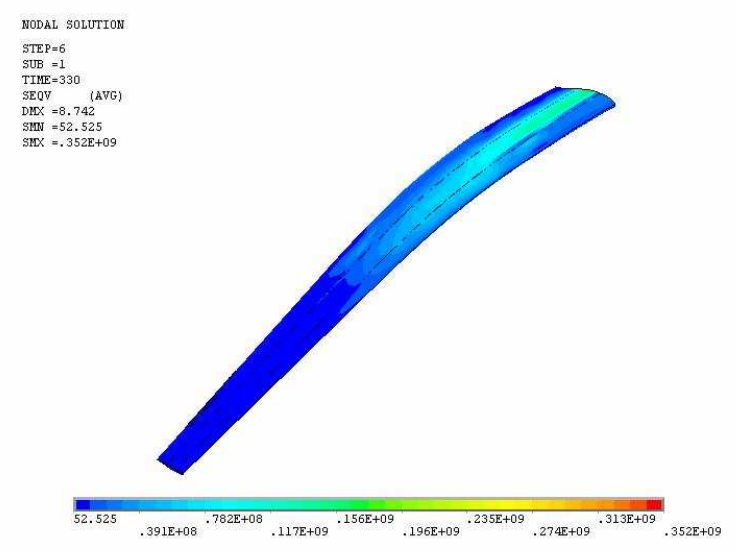

Fig. 10. Von Misses stresses in the fatigue analysis after $330 \mathrm{~s}$ of ciclyc loading.

Although not shown, the accumulated damage after one million of cycles of periodic loading is below the critical value of one. This fact is due to the low strength of the undergone stresses by the blade, around 0.4 times the ultimate stress.

\section{Conclusions}

This paper describes the aeromechanical evaluation of a large HAWT of rated power of $3 \mathrm{Mw}$, denominated $\mathrm{W} 15 \mathrm{~S}$, according to the configuration referred in [3]. In spite of the lack of information about the profiles aerodynamic characteristics, the aerodynamic behaviour of the blade is fairly good. The structural study comprised three analysis: static, dynamic and fatigue. As a result, the Safety Factor, which measures the structure quality under static loads, is below the critical value of one, so the blade will not break. The fatigue was evaluated with the Degradation Lineal Accumulation model which provided, after one million of cyclic loading, an accumulated damage below the critical value of one; therefore the blade will not fail due to fatigue.

\section{Acknowledgement}

The authors gratefully acknowledge the funding of the Vicerrectoría de Investigaciones y Desarrollo Tecnológico of Universidad Autónoma de Occidente through the project "Evaluación aerodinámica y 
estructural de aerogeneradores de eje horizontal de potencia nominal superior al megawatio".

\section{References}

[1] S. Laín, J.A. García and R. Aliod, "Development of a lifting surface-2D panel method to compute the threedimensional pressure distribution over the blade of a HAWT". Wind Engineering, vol. 19, pp.21-40. 1995.

[2] B. Quintero, M.F. Valle, H.E. Jaramillo, S. Laín, "Aeromechanical study of horizontal axis wind turbines" in Conf. Rec. 2004 CIUREE Int. Conf. Communications, pp. 63-69.

[3] K.J. Jackson, M.D. Zuteck, C.P. van Dam, K.J. Standish and D. Berry, "Innovative design approaches for large wind turbine blades". Wind Energy, vol. 8, pp. 141-171. 2005.

[4] W.Z. Stepniewsky and C.N. Keys, Rotary-wing aerodynamics, Dover 1984.

[5] J. Moran, An introduction to theoretical and computational aerodynamics. John Wiley \& Sons, New York, 1984.

[6] A. Palmgren, „Die Lebendauer von Kugellagern“. Verfahrenstechnik, vol. 68, pp. 339-341, 1924.

[7] M.A. Miner, "Cumulative damage in fatigue". J. Appl. Mech., vol. 12, pp. A159-A164, 1945. 